\title{
Universal spectral correlations of the Dirac operator at finite temperature
}

\author{
Thomas Guhr and Tilo Wettig \\ a Max-Planck-Institut für Kernphysik, Postfach 103980, D-69029 Heidelberg, \\ Germany \\ b Institut für Theoretische Physik, Technische Universität München, D-85747 \\ Garching, Germany
}

\begin{abstract}
Using the graded eigenvalue method and a recently computed extension of the Itzykson-Zuber integral to complex matrices, we compute the $k$-point spectral correlation functions of the Dirac operator in a chiral random matrix model with a deterministic diagonal matrix added. We obtain results both on the scale of the mean level spacing and on the microscopic scale. We find that the microscopic spectral correlations have the same functional form as at zero temperature, provided that the microscopic variables are rescaled by the temperature-dependent chiral condensate.
\end{abstract}

PACS: $05.45 .+\mathrm{b} ;$ 11.30.Rd; 12.38.Aw; 12.38.Lg

Keywords: Spectrum of the QCD Dirac operator; Chiral random matrix models; Finite temperature models

\section{Introduction}

In the past few years, random matrix models have started to be used in order to understand details of the spectral properties of the Dirac operator of non-abelian gauge theories [1 21]. Since random matrix models are free of dynamical input, one expects them to describe universal spectral properties, i.e., properties which do not depend on the specific details of the dynamics. In the case of non-relativistic quantum mechanics, it has long been known for a variety of physical systems that random matrix models are able to describe the universal spectral correlations on the scale of the mean level spacing [22 241. In the case of the Dirac operator of QCD the chiral structure has to be incorporated, requiring the introduction of the chiral random matrix ensembles [3]. The symmetry constraints lead to the definition of the chiral Gaussian 
Orthogonal (chGOE), Unitary (chGUE) and Symplectic (chGSE) Ensembles. A precise classification can be found in Ref. [7]. Our chiral random matrix model is based on unitary symmetry and will be defined in Sec. 2 .

For the case of QCD with two colors, the universality of the spectral correlations in the bulk of the spectrum of the lattice Dirac operator was demonstrated convincingly in Ref. [10] where predictions from random matrix theory were compared with lattice data obtained by Kalkreuter. However, the fact that the chiral symmetry of the Dirac operator is spontaneously broken implies that another type of universality exists concerning the microscopic limit of the spectral density. The spectral density is defined by

$$
\rho(\lambda)=\left\langle\sum_{n} \delta\left(\lambda-\lambda_{n}\right)\right\rangle_{A},
$$

where the $\lambda_{n}$ are the eigenvalues of the Dirac operator $i D=i \gamma_{\mu} \partial_{\mu}+\gamma_{\mu} A_{\mu}$ (in euclidean space) and the average is over all gauge field configurations $A$ weighted by the euclidean QCD action. Note that $i D$ is hermitian so that the $\lambda_{n}$ are real. Moreover, because $i D$ anticommutes with $\gamma_{5}$ all eigenvalues occur in pairs $\pm \lambda$ so that $\rho$ is an even function. The chiral condensate $\Xi$ is related to the spectral density via the Banks-Casher formula [25]

$$
\Xi=\frac{\pi}{V} \rho(0),
$$

where $V$ is the space-time volume. The microscopic spectral density is defined by [3]

$$
\rho_{s}(u)=\lim _{V \rightarrow \infty} \frac{1}{V \Xi} \rho\left(\frac{u}{V \Xi}\right) .
$$

Based on sum rules derived by Leutwyler and Smilga [26], $\rho_{s}$ was conjectured to be a universal quantity [3]. This conjecture was supported by several works [4, $60,13,19,27,29]$. As a universal function, the microscopic spectral density can be computed in the framework of random matrix models. This task was performed at zero temperature for all three chiral ensembles [ [ [6]. The most direct evidence for the universality of $\rho_{s}$ came from a recent comparison of lattice data obtained by Berbenni-Bitsch and Meyer with predictions from random matrix theory where impressive agreement was obtained [30].

Recently, the chiral random matrix models were extended to finite temperature $T$ and finite chemical potential $\mu$ [14 18]. This leads to the addition of a deterministic diagonal matrix to the random matrix used at zero $T$ and $\mu$. In such models, one can investigate, e.g., how the chiral condensate decreases as a function of $T$ and/or $\mu$. For the simplest model in which the additional diagonal matrix is given by the lowest Matsubara frequency $\pi T$ and, thus, proportional to the unit matrix, the spectral density has been computed in Refs. [16],19] and its microscopic limit in Ref. [19]. In the present paper, we 
(i) generalize these results to arbitrary diagonal matrices and (ii) compute all higher spectral correlation functions, both on the scale of the mean level spacing and in the microscopic limit. The first extension is important since it is often desirable to consider more general diagonal matrices, e.g., if one wants to include higher Matsubara frequencies or in the approach of Ref. [15] where the formation of instanton-anti-instanton molecules was modeled. The second extension allows for further analysis of lattice results for, e.g., scalar susceptibilities. Our results also add further evidence to the conjecture that the microscopic spectral correlations are universal.

This paper is organized as follows. In Sec. 2 we define the model and summarize some known results which will be refered to in the following. In Sec. 3 we set up the computation of the spectral correlation functions in the framework of the graded eigenvalue method and express the result in terms of a double integral. This double integral is evaluated in Sec. 1 for the spectral density and the spectral correlations on the scale of the mean level spacing and in Sec. 5 for the microscopic limit of the spectral correlation functions, respectively. The results are discussed in Sec. 6. Three appendices are provided to discuss convergence issues and technical details associated with saddle-point approximations in Sec. 5 .

\section{Formulation of the problem}

We formulate the problem in the framework of random matrix theory in Sec. 2.1. In Sec. 2.2, we discuss the connection with Dyson's Brownian motion model.

\subsection{Definition of the model}

We are interested in studying the spectral correlations of the Dirac operator on the scale of the mean level spacing and in the microscopic limit. Since the spectral correlations are universal in these two limits they can be obtained in the framework of a random matrix model [3]. In such a model, the matrix of the Dirac operator (in euclidean space) is replaced by a random matrix with appropriate symmetries, the average over gauge field configurations is replaced by the average over random matrices, and the complicated weight function containing the euclidean action is replaced by a simple Gaussian distribution of the random matrices. Moreover, at finite temperature a deterministic diagonal matrix with real entries has to be added to the random matrix of the Dirac operator [14 [16, 18]. (Note that one could also add a non-diagonal deterministic matrix since it could always be diagonalized by a suitable rotation 
of the basis states. This is possible due to the invariance of the random matrix ensembles with respect to basis changes.)

The matrix representing the Dirac operator in a chiral basis has the structure

$$
A=\left[\begin{array}{cc}
i m \mathbb{1}_{N} & W+Y \\
W^{\dagger}+Y & i m \mathbb{1}_{N}
\end{array}\right]
$$

where $m$ is an additional mass term, $W$ is a square random matrix of dimension $N$, and $Y=\operatorname{diag}\left(y_{1}, \ldots, y_{N}\right)$ is a diagonal matrix with real entries. The total number of eigenvalues of $A$ is $2 N$ which can be identified with the space-time volume $V$ in Eqs. (2) and (3). The specific form of $Y$ is model dependent, but we will obtain results for an arbitrary choice of $Y$. Note that we consider the quenched case where $N_{f}$, the number of flavors, is equal to zero. Furthermore, we consider only square matrices $W$. Using rectangular matrices one can model the topological charge $\nu$ which corresponds to the difference between the number of rows and columns of $W$. The extension of the present calculation to non-zero $N_{f}$ and $\nu$ is in progress.

In this paper, we study the chiral GUE appropriate for QCD with three or more colors for which $W$ is a complex matrix. The probability distribution of $W$ is a Gaussian,

$$
P(W)=\left(\frac{N \Sigma^{2}}{\pi}\right)^{N^{2}} \exp \left(-N \Sigma^{2} \operatorname{tr} W W^{\dagger}\right),
$$

where $\Sigma$ is a real parameter equal to the chiral condensate at zero temperature.

We shall consider separately the case where all the $y_{n}$ are equal, i.e., where $Y=y \mathbb{1}_{N}$ (this will be refered to as the "special case") and the case where $Y$ is arbitrary (this is the "general case"). This separation is useful since the various mathematical structures are most easily identified in the special case from which they can then be generalized.

The chiral condensate $\Xi$, which depends on the matrix $Y$, has already been computed for both the special case [14],

$$
\Xi=\Sigma \sqrt{1-(\Sigma y)^{2}}
$$

and the general case 15],

$$
\Xi=\Sigma^{2} \bar{x}
$$

Here, $\bar{x}$ is the only real and positive solution of

$$
\Sigma^{2}=\frac{1}{N} \sum_{n=1}^{N} \frac{1}{y_{n}^{2}+\bar{x}^{2}}
$$


or zero if no such solution exists. (See Appendix $\mathrm{C}$ for a proof that this equation has at most one real and positive solution.)

The eigenvalue density and its microscopic limit for the special case were computed in Ref. [19]. In the following, we will compute the eigenvalue density and the spectral correlation functions on the scale of the mean level spacing as well as the corresponding microscopic limits for the general case.

The $k$-point correlation functions measure the probability of finding energies around the points $x_{1}, \ldots, x_{k}$, regardless of labeling. Apart from trivial $\delta$-functions they are defined by

$$
R_{k}\left(x_{1}, \ldots, x_{k}\right)=\left(-\frac{1}{\pi}\right)^{k} \int d[W] P(W) \prod_{p=1}^{k} \operatorname{Im} \operatorname{tr} \frac{1}{x_{p}^{+}-A},
$$

where $x_{p}^{+}=x_{p}+i \varepsilon$ with $\varepsilon$ positive infinitesimal. In particular, we have $\rho(\lambda)=$ $R_{1}(\lambda)$. It is technically simpler to compute the functions

$$
\hat{R}_{k}\left(x_{1}, \ldots, x_{k}\right)=\left(-\frac{1}{\pi}\right)^{k} \int d[W] P(W) \prod_{p=1}^{k} \operatorname{tr} \frac{1}{x_{p}^{+}-A}
$$

from which the $R_{k}$ can be reconstructed by taking the imaginary parts of the traces.

\subsection{Joint probability density function of the radial coordinates}

Dyson's Brownian motion model of random matrix theory [23, 31] describes the transition from an arbitrary to a Gaussian ensemble. Since this approach is often used, we establish the link to our random matrix model. The main modification is of course due to the chiral structure. In Ref. [32], we gave a detailed discussion of a diffusion equation in the space of arbitrary complex ordinary and supermatrices. In the case of ordinary matrices, this diffusion is the chiral analogue for the chGUE of Dyson' Brownian motion for the GUE.

The pseudo-diagonalization of the matrix $W$ can be written as $W=U X \bar{V}$, where $U \in \mathrm{U}(N), \bar{V} \in \mathrm{U}(N) / \mathrm{U}^{N}(1)$, and $X=\operatorname{diag}\left(x_{1}, \ldots, x_{N}\right)$. The $x_{n}$ are referred to as radial coordinates, they are defined on the positive real axis. The transformation of the Cartesian volume element to radial and angular coordinates reads 32

$$
d[W]=\Delta_{N}^{2}\left(X^{2}\right) \prod_{n=1}^{N} x_{n} d x_{n} d \mu(U) d \mu(\bar{V}),
$$

where $\Delta_{N}\left(X^{2}\right)=\prod_{n<m}\left(x_{n}^{2}-x_{m}^{2}\right)$ is the Vandermonde determinant and $d \mu(U)$ and $d \mu(\bar{V})$ are the invariant Haar measures. 
Consider now $Y=0$ in Eqs. (4) and (9). Obviously, the integrand depends only on the radial coordinates and the group integrals are trivial. This socalled rotation invariance is broken for non-zero $Y$. Nevertheless, even in this case, the mathematical problem can be reduced to integrals over the radial coordinates. By shifting the real part of the diagonal of $W$ and thus also of $W^{\dagger}$ by the matrix $Y$, the breaking of the rotation invariance is removed from the traces in Eq. (9). The resulting probability density function $P(W-Y)$, however, now depends explicitly on the angular coordinates $U$ and $\bar{V}$. To reduce the problem to the radial coordinates, one has to perform the group integrations

$$
\Gamma(X, Y)=\int d \mu(U) \int d \mu(\bar{V}) P(U X \bar{V}-Y)
$$

which can be viewed as the extension of the Itzykson-Zuber integral to complex matrices. In Refs. [32,20], this integral was calculated,

$$
\Gamma(X, Y)=\frac{1}{N !} \frac{\operatorname{det}\left[\gamma\left(x_{n}, y_{m}\right)\right]_{n, m=1, \ldots, N}}{\Delta_{N}\left(X^{2}\right) \Delta_{N}\left(Y^{2}\right)}
$$

where the entries of the determinant are given by the functions

$$
\gamma\left(x_{n}, y_{m}\right)=2 N \Sigma^{2} \exp \left(-N \Sigma^{2}\left(x_{n}^{2}+y_{m}^{2}\right)\right) I_{0}\left(2 N \Sigma^{2} x_{n} y_{m}\right) .
$$

Here, $I_{0}(z)$ is the modified Bessel function of zeroth order.

Collecting everything, the $k$-level correlation function is obtained by integrating the distribution

$$
\Gamma(X, Y) \Delta_{N}^{2}\left(X^{2}\right) \prod_{n=1}^{N} x_{n}=\frac{1}{N !} \operatorname{det}\left[\gamma\left(x_{n}, y_{m}\right)\right]_{n, m=1, \ldots, N} \frac{\Delta_{N}\left(X^{2}\right)}{\Delta_{N}\left(Y^{2}\right)} \prod_{n=1}^{N} x_{n}
$$

over the $N-k$ radial coordinates $x_{k+1}, \ldots, x_{N}$. The expression (15) is called the joint probability density function. Thus, we have identified the kernel of the diffusion equation of Ref. [32] with the joint probability density function of our random matrix model. Apparently, it is highly non-trivial to actually perform the integrations over the $N-k$ radial coordinates. In Sec. 3.2, we will show that the supersymmetric analogue of the joint probability density function can be used for a much faster and explicit calculation of the spectral correlation functions.

\section{Correlation functions and supersymmetry}

After the introduction of random matrix theory by Wigner [33], many of the mathematical details were worked out by Dyson [31] and Mehta [23]. In 1983, 
Efetov introduced the supersymmetry method [34] which was further developed in Ref. 35]. In Sec. 3.1 we employ these methods to construct a supersymmetric representation for the spectral correlation functions. It turns out that the formal aspects of the problem discussed here are related to the transition from Poisson regularity to the GUE. In Ref. [36], this transition was discussed with supersymmetry by extending the graded eigenvalue method developed in Ref. [37]. In Sec. 3.2 we further generalize this approach to the chGUE. The main ingredient is now the supersymmetric extension of the Itzykson-Zuber like integral [32] mentioned in the previous section.

\subsection{Supersymmetric representation}

The correlation functions (9) and (10) can be obtained from the generating function

$$
Z_{k}(J)=\int d[W] P(W) \frac{\prod_{p=1}^{k} \operatorname{det}\left(x_{p}^{+}+J_{p}-A\right)}{\prod_{p=1}^{k} \operatorname{det}\left(x_{p}^{+}-J_{p}-A\right)},
$$

where $J$ stands for $J_{1}, \ldots, J_{k}$. Note that $Z_{k}(0)=1$. The $\hat{R}_{k}$ are then generated by

$$
\hat{R}_{k}\left(x_{1}, \ldots, x_{k}\right)=\left.\left(-\frac{1}{2 \pi}\right)^{k} \frac{\partial^{k}}{\prod_{p=1}^{k} \partial J_{p}} Z_{k}(J)\right|_{J_{p}=0}
$$

The ratio of the products of determinants in Eq. (16) can be written as an inverse graded determinant which, in turn, can be expressed as an integral over graded vectors. We define $N$-dimensional vectors $z_{p 1}, z_{p 2}, \zeta_{p 1}, \zeta_{p 2}$, and $2 N$-dimensional vectors $z_{p}=\left(z_{p 1}, z_{p 2}\right)^{T}$ and $\zeta_{p}=\left(\zeta_{p 1}, \zeta_{p 2}\right)^{T}$. The $z_{p}$ contain commuting variables whereas the $\zeta_{p}$ contain anticommuting variables. These variables are combined in a graded vector $\psi=\left(z_{1}, \ldots, z_{k}, \zeta_{1}, \ldots, \zeta_{k}\right)^{T}$ of dimension $4 k N$. The generating function (16) can then be written as

$$
\begin{aligned}
Z_{k}(J) & =\int d[W] P(W) \operatorname{detg}^{-1} D \\
& =\int d[W] P(W) \int d[\psi] \exp \left(i \psi^{\dagger} D \psi\right),
\end{aligned}
$$

where $D=\left(x^{+}+J\right) \otimes \mathbb{1}_{2 N}-\mathbb{1}_{2 k} \otimes A$ is a matrix of dimension $4 k N$. Here, $x+J=\operatorname{diag}\left(x_{1}-J_{1}, \ldots, x_{k}-J_{k}, x_{1}+J_{1}, \ldots, x_{k}+J_{k}\right)$ is a graded matrix of dimension $2 k$, and our convention for the direct product of two matrices $S$ and $T$ with number of rows $r_{S}, r_{T}$ and number of columns $c_{S}, c_{T}$ is that $(S \otimes T)_{(i-1) r_{T}+k,(j-1) c_{T}+l}=S_{i j} T_{k l}$. The integration over $W$ can now be per- 
formed. We write

$$
\psi^{\dagger} \mathbb{1}_{2 k} \otimes\left[\begin{array}{cc}
0 & W \\
W^{\dagger} & 0
\end{array}\right] \psi=\operatorname{tr}\left(W E+W^{\dagger} E^{\dagger}\right)
$$

with

$$
E=\sum_{p=1}^{k}\left(z_{p 2} z_{p 1}^{\dagger}-\zeta_{p 2} \zeta_{p 1}^{\dagger}\right)
$$

and obtain the ensemble average

$$
\begin{aligned}
\int d[W] & P(W) \exp \left(-i \operatorname{tr}\left(W E+W^{\dagger} E^{\dagger}\right)\right) \\
= & \exp \left(-\frac{1}{N \Sigma^{2}} \operatorname{tr} E E^{\dagger}\right)=\exp \left(-\frac{1}{N \Sigma^{2}} \operatorname{trg} F_{1} F_{2}\right) .
\end{aligned}
$$

The $F_{i}(i=1,2)$ are $2 k$-dimensional graded matrices of the structure

$$
F_{i}=\left[\begin{array}{ll}
F_{i}^{B B} & F_{i}^{B F} \\
F_{i}^{F B} & F_{i}^{F F}
\end{array}\right],
$$

where the boson-boson and fermion-fermion block both have dimension $k$. The entries are given by scalar products

$$
\begin{array}{ll}
\left(F_{i}^{B B}\right)_{p p^{\prime}}=z_{p i}^{\dagger} z_{p^{\prime} i} & \left(F_{i}^{B F}\right)_{p p^{\prime}}=\zeta_{p i}^{\dagger} z_{p^{\prime} i} \\
\left(F_{i}^{F B}\right)_{p p^{\prime}}=z_{p i}^{\dagger} \zeta_{p^{\prime} i} & \left(F_{i}^{F F}\right)_{p p^{\prime}}=\zeta_{p i}^{\dagger} \zeta_{p^{\prime} i}
\end{array}
$$

with $p, p^{\prime}=1, \ldots, k$. We now perform a Hubbard-Stratonovitch transformation to decouple $F_{1}$ and $F_{2}$,

$$
\begin{aligned}
\exp & \left(-\frac{1}{N \Sigma^{2}} \operatorname{trg} F_{1} F_{2}\right) \\
& =2^{2 k^{2}} \int d[\sigma] \exp \left(-N \Sigma^{2} \operatorname{trg} \sigma \sigma^{\dagger}+i \operatorname{trg}\left(F_{1} \sigma+F_{2} \sigma^{\dagger}\right)\right)
\end{aligned}
$$

where $\sigma$ is a complex graded matrix of the same structure as $F_{i}$. Combining Eqs. (18), (21), and (24), we obtain

$$
Z_{k}(J)=2^{2 k^{2}} \int d[\psi] \int d[\sigma] \exp \left(-N \Sigma^{2} \operatorname{trg} \sigma \sigma^{\dagger}+i \psi^{\dagger} G \psi\right)
$$

with

$G=\sigma \otimes\left[\begin{array}{cc}\mathbb{1}_{N} & 0 \\ 0 & 0\end{array}\right]+\sigma^{\dagger} \otimes\left[\begin{array}{cc}0 & 0 \\ 0 & \mathbb{1}_{N}\end{array}\right]+\left(x^{+}+J\right) \otimes \mathbb{1}_{2 N}-\mathbb{1}_{2 k} \otimes\left[\begin{array}{cc}0 & Y \\ Y & 0\end{array}\right]$. 
Note that we have set the quark mass $m$ equal to zero in (26). It can always be reinstated at the end of the calculation if we shift the $x_{p}$ by $\mathrm{im}$.

We would now like to interchange the order of the $\sigma$-and the $\psi$-integrations in (25) and to perform the integration over $\psi$. This would be allowed if the $\psi$ integral were uniformly convergent. Uniform convergence is guaranteed for the ordinary GUE but not for the chGUE. In fact, the $\psi$-integral as it stands is not uniformly convergent. This presents certain problems which are discussed in some detail in Appendix A. There are various ways to proceed. For the present calculation, we note that there is a very strong conjecture that ignoring the convergence problem will lead to a result whose real part is incorrect but whose imaginary part is correct. Since we are only interested in the spectral correlation functions which are given by the imaginary part, we choose to go ahead with the calculation without modifications. However, the reader should consult Appendix $\mathrm{A}$ for a more complete discussion of this problem.

Ignoring the convergence issue, we now shift $\sigma$ and $\sigma^{\dagger}$ by $-(x+J)$ and perform the integration over $\psi$ to obtain after some algebra

$$
\begin{gathered}
Z_{k}(J)=2^{2 k^{2}} \int d[\sigma] \exp \left(-N \Sigma^{2} \operatorname{trg}(\sigma-(x+J))\left(\sigma^{\dagger}-(x+J)\right)\right) \\
\times \operatorname{detg}^{-1}\left(\sigma^{+}\left(\sigma^{+}\right)^{\dagger} \otimes \mathbb{1}_{N}-\mathbb{1}_{2 k} \otimes Y^{2}\right)
\end{gathered}
$$

In order to work out the integrals over the supermatrix, there are various ways to proceed. In particular, Efetov's 34 coset method has been used in numerous applications. It is based on a saddle-point approximation in the large- $N$ limit. To solve the problems outlined in the introduction we use the graded eigenvalue method [37,36] which is, in this context, particularly well suited.

\subsection{Reduction to eigenvalue integrals}

The matrix $\sigma$ can be written in pseudo-diagonal form as $\sigma=u s \bar{v}$, where $u \in$ $\mathrm{U}(k / k), \bar{v} \in \mathrm{U}(k / k) / \mathrm{U}^{2 k}(1)$, and $s=\operatorname{diag}\left(s_{1}, i s_{2}\right)$ with $s_{j}=\operatorname{diag}\left(s_{1 j}, \ldots, s_{k j}\right)$ for $j=1,2$ [32]. The $s_{p j}$ are real and non-negative. The transformation of the Cartesian volume element to radial and angular coordinates reads [32]

$$
\begin{aligned}
d[\sigma] & =J(s) d[s] d \mu(u) d \mu(\bar{v}) \\
d[s] & =\prod_{p=1}^{k} d s_{p 1} d s_{p 2} \\
J(s) & =B_{k}^{2}\left(s^{2}\right) \prod_{p=1}^{k} s_{p 1} s_{p 2}
\end{aligned}
$$


with

$$
\begin{aligned}
B_{k}(s) & =\frac{\Delta_{k}\left(s_{1}\right) \Delta_{k}\left(i s_{2}\right)}{\prod_{p, q}\left(s_{p 1}-i s_{q 2}\right)} \\
\Delta_{k}(x) & =\prod_{p<q}\left(x_{p}-x_{q}\right) .
\end{aligned}
$$

The group measure is the invariant Haar measure. We obtain

$$
\begin{aligned}
Z_{k}(J)= & 2^{2 k^{2}} \exp \left(-N \Sigma^{2} \operatorname{trg}(x+J)^{2}\right) \\
& \times \int d[s] J(s) \exp \left(-N \Sigma^{2} \operatorname{trg} s^{2}\right) \operatorname{detg}^{-1}\left(\left(s^{+}\right)^{2} \otimes \mathbb{1}_{N}-\mathbb{1}_{2 k} \otimes Y^{2}\right) \\
& \times \int d \mu(u) \int d \mu(\bar{v}) \exp \left(N \Sigma^{2} \operatorname{trg}\left(\sigma+\sigma^{\dagger}\right)(x+J)\right) .
\end{aligned}
$$

The integral over the diagonalizing groups has been computed in Ref. [32]. The result is

$$
\begin{aligned}
\int & d \mu(u) \int d \mu(\bar{v}) \exp \left(N \Sigma^{2} \operatorname{trg}\left(\sigma+\sigma^{\dagger}\right)(x+J)\right) \\
= & \frac{\left(2 N \Sigma^{2}\right)^{2 k}}{2^{2 k^{2}}(k !)^{2}} \frac{\operatorname{det}\left[I_{0}\left(2 N \Sigma^{2} s_{p 1}\left(x_{p^{\prime}}-J_{p^{\prime}}\right)\right)\right] \operatorname{det}\left[J_{0}\left(2 N \Sigma^{2} s_{q 2}\left(x_{q^{\prime}}+J_{q^{\prime}}\right)\right)\right]}{B_{k}\left(s^{2}\right) B_{k}\left((x+J)^{2}\right)}
\end{aligned}
$$

with $p, p^{\prime}=1, \ldots, k$ and $q, q^{\prime}=1, \ldots, k$. Here, $J_{0}$ and $I_{0}$ are the Bessel and modified Bessel functions of order 0, respectively. Thus, Eq. (31) becomes

$$
\begin{aligned}
Z_{k}(J)= & 1+\frac{\left(2 N \Sigma^{2}\right)^{2 k} \exp \left(-N \Sigma^{2} \operatorname{trg}(x+J)^{2}\right)}{(k !)^{2} B_{k}\left((x+J)^{2}\right)} \int d[s] \prod_{p=1}^{k} s_{p 1} s_{p 2} B_{k}\left(s^{2}\right) \\
& \times \exp \left(-N \Sigma^{2} \operatorname{trg} s^{2}\right) \operatorname{detg}^{-1}\left(\left(s^{+}\right)^{2} \otimes \mathbb{1}_{N}-\mathbb{1}_{2 k} \otimes Y^{2}\right) \\
& \times \operatorname{det}\left[I_{0}\left(2 N \Sigma^{2} s_{p 1}\left(x_{p^{\prime}}-J_{p^{\prime}}\right)\right)\right]_{p, p^{\prime}=1, \ldots, k} \\
& \times \operatorname{det}\left[J_{0}\left(2 N \Sigma^{2} s_{q 2}\left(x_{q^{\prime}}+J_{q^{\prime}}\right)\right)\right]_{q, q^{\prime}=1, \ldots, k}
\end{aligned}
$$

Note the contribution of unity to the generating function. It ensures the correct normalization of $Z_{k}$ at $J=0$ and is due to so-called Efetov-Wegner terms which were discussed in detail in Ref. [32.

The calculation now greatly simplifies due to the determinant structure of the function $B_{k}(s)$. In fact, Eq. (29) can be rewritten as

$$
B_{k}(s)=\operatorname{det}\left[\frac{1}{s_{p 1}-i s_{q 2}}\right]_{p, q=1, \ldots, k} .
$$


Performing the differentiations with respect to the $J_{p}$ according to Eq. (17) and using the determinant structure of $Z_{k}$, straightforward manipulations show that the $k$-point functions can be written as

$$
R_{k}\left(x_{1}, \ldots, x_{k}\right)=\operatorname{det}\left[C_{N}\left(x_{p}, x_{q}\right)\right]_{p, q=1, \ldots, k},
$$

where the function $C_{N}$ is given by

$$
\begin{aligned}
C_{N}\left(x_{p}, x_{q}\right)= & \frac{2}{\pi}\left(2 N \Sigma^{2}\right)^{2} x_{p} \int_{0}^{\infty} \int_{0}^{\infty} d s_{1} d s_{2} \frac{s_{1} s_{2}}{s_{1}^{2}+s_{2}^{2}} \exp \left(-N \Sigma^{2}\left(s_{1}^{2}+s_{2}^{2}\right)\right) \\
& \times I_{0}\left(2 N \Sigma^{2} x_{p} s_{1}\right) J_{0}\left(2 N \Sigma^{2} x_{q} s_{2}\right) \operatorname{Im} \prod_{n=1}^{N} \frac{y_{n}^{2}+s_{2}^{2}}{y_{n}^{2}-\left(s_{1}+i \varepsilon\right)^{2}} .
\end{aligned}
$$

Hence, all $k$-point functions are known if the double integral (36) can be computed. This can be done by saddle-point approximation in the large- $N$ limit. However, for some applications it is more convenient to rewrite the double integral as an integral over a $2 \times 2$ complex graded matrix $\sigma$ by using our result (32) for the integral over the diagonalizing groups in the opposite direction. This is by no means a trivial step since we needed the angular integral in order to obtain the determinant structure of (35). Using Eq. (32) for $k=1$, we obtain from (36)

$$
\begin{aligned}
& C_{N}\left(x_{p}, x_{q}\right)=\frac{8}{\pi} x_{p} \frac{e^{N \Sigma^{2}\left(x_{p}^{2}-x_{q}^{2}\right)}}{x_{p}^{2}-x_{q}^{2}} \operatorname{Im} \int d[\sigma] \exp \left(-N \mathcal{L}\left(\sigma, \sigma^{\dagger}\right)\right) \\
& \mathcal{L}\left(\sigma, \sigma^{\dagger}\right)=\Sigma^{2} \operatorname{trg} \sigma \sigma^{\dagger}-\frac{1}{N} \operatorname{trg} \log \left(\left(\sigma^{+}+x\right)\left(\left(\sigma^{+}\right)^{\dagger}+x\right) \otimes \mathbb{1}_{N}-\mathbb{1}_{2} \otimes Y^{2}\right),
\end{aligned}
$$

where $\sigma$ is now a $2 \times 2$ complex graded matrix and $x=\operatorname{diag}\left(x_{p}, x_{q}\right)$. Note that $\sigma$ and $\sigma^{\dagger}$ have been shifted by $x$ to obtain Eqs. (37) and (38). The integral in Eq. (37) can also be computed in saddle-point approximation in the large- $N$ limit. Since the matrix $\sigma$ can be pseudo-diagonalized, $\sigma=u s \bar{v}$, we can assume that $\sigma$ is diagonal at the saddle point, $\bar{\sigma}=\operatorname{diag}\left(s_{1}, i s_{2}\right)$, and thus obtain from (38)

$$
\mathcal{L}\left(s_{1}, i s_{2}\right)=\Sigma^{2}\left(s_{1}^{2}+s_{2}^{2}\right)-\frac{1}{N} \sum_{n=1}^{N} \log \frac{\left(i s_{2}+x_{q}\right)^{2}-y_{n}^{2}}{\left(s_{1}+x_{p}\right)^{2}-y_{n}^{2}},
$$

where we have omitted the imaginary increments.

We will use Eq. (37) to compute the spectral density and the universal spectral correlations in the bulk of the spectrum in Sec. 1 and Eq. (36) to compute the microscopic limits of the spectral density and the spectral correlation 
functions in Sec. 5. Our expressions allow for a relatively straightforward finite$N$ analysis. However, we are eventually interested in the thermodynamic limit. Therefore, we compute our results in the limit $N \rightarrow \infty$.

\section{Spectral density and universal spectral correlations in the bulk of the spectrum}

In this section, we compute the spectral density and the universal spectral correlations in the bulk of the spectrum on the scale of the mean level spacing, for both the special and the general case. The mean level spacing $D(x)$ is defined as $D(x)=1 / \rho(x)$. With our definitions, we have $D(x) \sim 1 /(N \Sigma)$ in

the bulk of the spectrum. Thus, the correlations are to be computed for energy differences $\sim 1 /(N \Sigma)$. We discuss the special case in Sec. 4.1 and turn to the general case in Sec. 4.2.

\subsection{Special case}

In the special case $Y=y \mathbb{1}_{N}$ with $y$ real, $(39)$ becomes

$$
\mathcal{L}\left(s_{1}, i s_{2}\right)=\Sigma^{2}\left(s_{1}^{2}+s_{2}^{2}\right)-\log \frac{\left(i s_{2}+x_{q}\right)^{2}-y^{2}}{\left(s_{1}+x_{p}\right)^{2}-y^{2}} .
$$

To minimize $\mathcal{L}$, we differentiate, first with respect to $s_{1}$,

$$
\frac{\partial \mathcal{L}}{\partial s_{1}}=2 \Sigma^{2} s_{1}+\frac{2\left(s_{1}+x_{p}\right)}{\left(s_{1}+x_{p}\right)^{2}-y^{2}} \stackrel{!}{=} 0
$$

which yields a cubic equation for $s_{1}$. Differentiation of $\mathcal{L}$ with respect to $i s_{2}$ yields an identical equation with $s_{1}$ and $x_{p}$ replaced by $i s_{2}$ and $x_{q}$, respectively. To compute the spectral density, we have to take the limit $x_{p}=x_{q}$. To compute the universal spectral correlations, $x_{p}-x_{q}$ is of order $1 /(N \Sigma)$ and, thus, $x_{p}=x_{q}$ in the large- $N$ limit. Hence, the saddle-point equations yield identical solutions for $s_{1}$ and $i s_{2}$, and we define $s_{1}=i s_{2} \equiv \bar{s}$ at the saddle point. Furthermore, we define $x=\left(x_{p}+x_{q}\right) / 2$ and $\Delta x=x_{p}-x_{q}$. Note that $x_{p}=x_{q}=x$ for $N \rightarrow \infty$. The saddle-point equation then reads

$$
\bar{s}^{3}+2 \bar{s}^{2} x+\bar{s}\left(1 / \Sigma^{2}+x^{2}-y^{2}\right)+x / \Sigma^{2}=0 .
$$

The Lagrangian (40) at the saddle point can then be expanded in $\Delta x$,

$$
\mathcal{L}_{0}=-\log \frac{(\bar{s}+x-\Delta x / 2)^{2}-y^{2}}{(\bar{s}+x+\Delta x / 2)^{2}-y^{2}} \approx \frac{2(\bar{s}+x)}{(\bar{s}+x)-y^{2}} \Delta x=-2 \Sigma^{2} \bar{s} \Delta x,
$$


where we have used Eq. (41) in the last step. The integral over the quadratic fluctuations yields a factor of $1 / 4$ (cf. our convention for the normalization of the Gaussian in Eq. (24)), and we obtain from Eq. (37)

$$
\begin{aligned}
C_{N}\left(x_{p}, x_{q}\right) & =\frac{8}{\pi} x_{p} \frac{e^{N \Sigma^{2}\left(x_{p}^{2}-x_{q}^{2}\right)}}{x_{p}^{2}-x_{q}^{2}} \frac{1}{4} \operatorname{Im} \exp \left(-N \mathcal{L}_{0}\right) \\
& \approx \frac{e^{2 N \Sigma^{2} x \Delta x}}{\pi \Delta x} \operatorname{Im} \exp \left(2 N \Sigma^{2} \bar{s} \Delta x\right) \\
& =\frac{\sin \left(2 N \Sigma^{2} \Delta x \operatorname{Im} \bar{s}\right)}{\pi \Delta x} \exp \left(2 N \Sigma^{2}(x+\operatorname{Re} \bar{s}) \Delta x\right)
\end{aligned}
$$

Because of the identity

$$
\operatorname{det}\left[f\left(x_{p}, x_{q}\right) \exp \left(\alpha\left(x_{p}-x_{q}\right)\right)\right]_{p, q=1, \ldots, k}=\operatorname{det}\left[f\left(x_{p}, x_{q}\right)\right]_{p, q=1, \ldots, k}
$$

for arbitrary $f$ and $\alpha$ we obtain in the large- $N$ limit

$$
R_{k}(x)=\operatorname{det}\left[K_{N}\left(x_{p}, x_{q}\right)\right]_{p, q=1, \ldots, k}
$$

with

$$
K_{N}\left(x_{p}, x_{q}\right)=\frac{\sin \left(2 N \Sigma^{2}\left(x_{p}-x_{q}\right) \operatorname{Im} \bar{s}\right)}{\pi\left(x_{p}-x_{q}\right)} .
$$

The spectral density now follows immediately,

$$
\rho(x)=K_{N}(x, x)=\frac{2 N \Sigma^{2}}{\pi} \operatorname{Im} \bar{s} .
$$

The cubic equation (42) always has one real solution. If the other two solutions are real, $K_{N}\left(x_{p}, x_{q}\right)$ and $\rho(x)$ are zero for this value of $x$. Otherwise, the other two solutions are complex conjugates of each other, and we have to take the solution with positive imaginary part because of the positive imaginary increment of $s$. This is the solution of (42) which enters (47) and (48).

Furthermore, we obtain from (48)

$$
D(x)=\frac{\pi}{2 N \Sigma^{2} \operatorname{Im} \bar{s}}
$$

so that (47) can be rewritten as

$$
D(x) K_{N}\left(x_{p}, x_{q}\right)=\frac{\sin \left(\pi\left(x_{p}-x_{q}\right) / D(x)\right)}{\pi\left(x_{p}-x_{q}\right) / D(x)} .
$$

Eq. (50) reproduces the well-known result for the spectral correlations on the scale of the mean level spacing. Thus, neither the chiral structure of our 
random matrix model nor the addition of the diagonal matrix $Y$ changes these universal correlations.

We would like to emphasize that the result for $\rho(\lambda)$ has been obtained previously in a simpler way $16,19,18$. However, our method has the virtue of also yielding all higher-order correlations and their microscopic limits in one single formalism.

\subsection{General case}

Consider now the case of general (but real) $Y$ where the Lagrangian has the form (39). Again, we minimize $\mathcal{L}$ and obtain identical solutions for $s_{1}$ and $i s_{2}$ in the limit $x_{p}=x_{q}$. With the definitions of the previous subsection, the equation for $\bar{s}$ is now

$$
\Sigma^{2} \bar{s}+\frac{1}{N} \sum_{n=1}^{N} \frac{\bar{s}+x}{(\bar{s}+x)^{2}-y_{n}^{2}}=0 .
$$

This is a polynomial equation of order $2 N+1$. We now demonstrate that this equation always has at least $2 N-1$ real solutions. The remaining two solutions are either real or complex conjugates of each other.

Define $z=\bar{s}+x$ and consider the function

$$
f(z)=\Sigma^{2}(z-x)+\frac{1}{N} \sum_{n=1}^{N} \frac{z}{z^{2}-y_{n}^{2}} .
$$

First assume that all the $y_{n}$ are different and non-zero. The function $f(z)$ has $2 N$ poles at $\pm y_{n}(n=1, \ldots, N)$. Now consider two adjacent poles $z_{m}<z_{m+1}$. We have $f\left(z_{m}+\varepsilon\right)>0$ and $f\left(z_{m+1}-\varepsilon\right)<0$, where $\varepsilon$ is positive infinitesimal. Since $f(z)$ is continuous for $z_{m}<z<z_{m+1}$, there must be a zero of $f$ in this interval. There are $2 N-1$ such intervals, hence there are $2 N-1$ real zeros of $f$. This proves our assertion. If some of the $y_{n}$ are equal or zero, the saddle-point equation (51) is of correspondingly lower order, and our argument applies as well.

In analogy to the result of the previous subsection, only the (single) saddle point with positive imaginary part contributes to $C_{N}\left(x_{p}, x_{q}\right)$. Expanding the Lagrangian about this saddle point yields

$$
\begin{aligned}
\mathcal{L}_{0} & =-\frac{1}{N} \sum_{n=1}^{N} \log \frac{(\bar{s}+x-\Delta x / 2)^{2}-y_{n}^{2}}{(\bar{s}+x+\Delta x / 2)^{2}-y_{n}^{2}} \approx \frac{2}{N} \sum_{n=1}^{N} \frac{(\bar{s}+x)}{(\bar{s}+x)-y_{n}^{2}} \Delta x \\
& =-2 \Sigma^{2} \bar{s} \Delta x
\end{aligned}
$$


using (51) in the last step. Going through the same steps as in the previous subsection, we obtain the same results as before, the only difference being that $s$ is now the only solution with positive imaginary part (or zero) of the more complicated equation (51) instead of the cubic equation (42).

\section{Microscopic limit}

In this section, we compute the $k$-point functions in the microscopic limit, i.e., for $x_{p} \sim 1 /(N \Sigma)$. In Sec. 5.1, we separate the radial integrals in (36), then consider the special case $Y=y \mathbb{1}_{N}$ in Sec. 5.2, and finally turn to the general case in Sec. 5.3. Although the special case obviously follows from the general case, we find it useful to discuss the special case first in order to make the structure of the radial integrals more explicit. The final result will be that the $k$-point functions are given by the zero-temperature result provided that the microscopic variables are rescaled by the temperature-dependent chiral condensate.

\subsection{Separation of the radial integrals}

We first define microscopic variables $u_{p}=2 N x_{p}$ and $u_{q}=2 N x_{q}$. The two integrals in Eq. (36) can be decoupled by writing

$$
\frac{\exp \left(-N \Sigma^{2}\left(s_{1}^{2}+s_{2}^{2}\right)\right)}{s_{1}^{2}+s_{2}^{2}}=N \Sigma^{2} \int_{1}^{\infty} d t \exp \left(-N \Sigma^{2} t\left(s_{1}^{2}+s_{2}^{2}\right)\right) .
$$

We thus obtain

$$
C_{N}\left(u_{p}, u_{q}\right)=\frac{4 N^{2} \Sigma^{6}}{\pi} u_{p} \int_{1}^{\infty} d t f_{1}(t) f_{2}(t)
$$

with

$$
\begin{aligned}
& f_{1}(t)=\int_{0}^{\infty} d s_{1} s_{1} \exp \left(-N \Sigma^{2} t s_{1}^{2}\right) I_{0}\left(\Sigma^{2} u_{p} s_{1}\right) \operatorname{Im} \prod_{n=1}^{N} \frac{1}{y_{n}^{2}-\left(s_{1}+i \varepsilon\right)^{2}} \\
& f_{2}(t)=\int_{0}^{\infty} d s_{2} s_{2} \exp \left(-N \Sigma^{2} t s_{2}^{2}\right) J_{0}\left(\Sigma^{2} u_{q} s_{2}\right) \prod_{n=1}^{N}\left(y_{n}^{2}+s_{2}^{2}\right) .
\end{aligned}
$$

These two integrals can now be computed in saddle-point approximation. 


\subsection{Special case}

Consider the special case $Y=y \mathbb{1}_{N}$ with $y$ real. The critical value of $y$ above which the chiral condensate vanishes is given by $y_{c}=1 / \Sigma$ so that we restrict ourselves to the interval $0 \leq y \leq y_{c}$ in the following. Eqs. (56) and (57) become

$$
\begin{aligned}
f_{1}(t) & =\int_{0}^{\infty} d s_{1} s_{1} \exp \left(-N \Sigma^{2} t s_{1}^{2}\right) I_{0}\left(\Sigma^{2} u_{p} s_{1}\right) \operatorname{Im} \frac{1}{\left(y^{2}-\left(s_{1}+i \varepsilon\right)^{2}\right)^{N}} \\
f_{2}(t) & =\int_{0}^{\infty} d s_{2} s_{2} \exp \left(-N \Sigma^{2} t s_{2}^{2}\right) J_{0}\left(\Sigma^{2} u_{q} s_{2}\right)\left(y^{2}+s_{2}^{2}\right)^{N} .
\end{aligned}
$$

These two integrals can be evaluated in saddle-point approximation in the large- $N$ limit. This somewhat technical task is performed in Appendix B. It turns out that depending on the value of $t$, the product of $f_{1}(t)$ and $f_{2}(t)$ can be of different order in $1 / N$. Specifically, there is a "critical" value of $t$,

$$
t_{c}=\frac{1}{(\Sigma y)^{2}}
$$

below which $f_{1}(t) f_{2}(t)$ is of order $1 / N$ and above which $f_{1}(t) f_{2}(t)$ is suppressed exponentially. Note that $t_{c} \geq 1$ since $y \leq y_{c}$. Thus, only the interval $1 \leq t \leq t_{c}$ contributes to the integral in Eq. (55) in leading order in $1 / N$. We obtain from Eqs. (B.8) and (B.15)

$$
f_{1}(t) f_{2}(t)=\frac{\pi}{4 N \Sigma^{4} t^{2}} J_{0}\left(\Sigma u_{p} \sqrt{1 / t-1 / t_{c}}\right) J_{0}\left(\Sigma u_{q} \sqrt{1 / t-1 / t_{c}}\right)
$$

to leading order in $1 / N$ in this region of $t$. Substituting $z=\Sigma \sqrt{1 / t-1 / t_{c}}$ and using $\Xi=\Sigma \sqrt{1-1 / t_{c}}$, we have from Eq. (55)

$$
\begin{aligned}
C_{N}\left(u_{p}, u_{q}\right) & =2 N u_{p} \int_{0}^{\Xi} d z z J_{0}\left(u_{p} z\right) J_{0}\left(u_{q} z\right) \\
& =2 N \Xi \tilde{u}_{p} \frac{\tilde{u}_{p} J_{1}\left(\tilde{u}_{p}\right) J_{0}\left(\tilde{u}_{q}\right)-\tilde{u}_{q} J_{0}\left(\tilde{u}_{p}\right) J_{1}\left(\tilde{u}_{q}\right)}{\tilde{u}_{p}^{2}-\tilde{u}_{q}^{2}}
\end{aligned}
$$

where we have defined $\tilde{u}_{p}=\Xi u_{p}$ and $\tilde{u}_{q}=\Xi u_{q}$ and used Eq. (11.3.29) of Ref. [38. In particular, we obtain for $k=1,2$

$$
\rho_{s}(\tilde{u})=\frac{\tilde{u}}{2}\left(J_{0}^{2}(\tilde{u})+J_{1}^{2}(\tilde{u})\right)
$$




$$
\rho_{s}\left(\tilde{u}_{1}, \tilde{u}_{2}\right)=\tilde{u}_{1} \tilde{u}_{2}\left(\frac{\tilde{u}_{1} J_{1}\left(\tilde{u}_{1}\right) J_{0}\left(\tilde{u}_{2}\right)-\tilde{u}_{2} J_{0}\left(\tilde{u}_{1}\right) J_{1}\left(\tilde{u}_{2}\right)}{\tilde{u}_{1}^{2}-\tilde{u}_{2}^{2}}\right)^{2}
$$

where we have subtracted the disconnected part of the two-point function in (65). Eq. (64) is in agreement with the result of Ref. [19]. The zero-temperature limit of Eq. (65) agrees with the result obtained in Ref. [4].

\subsection{General case}

We now generalize the results of the previous subsection to the case where $Y=$ $\operatorname{diag}\left(y_{1}, \ldots, y_{N}\right)$. We only require that the $y_{n}$ be real. The chiral condensate for this case has been computed in Ref. [15], and the condition for the existence of a condensate was found to be

$$
\Sigma^{2} \leq \frac{1}{N} \sum_{n=1}^{N} \frac{1}{y_{n}^{2}}
$$

Note that there is always a condensate if some of the $y_{n}$ are zero (or more precisely, if a finite fraction of the $y_{n}$ is zero). We shall restrict ourselves to choices of $Y$ for which the above condition is satisfied.

We are now dealing directly with the two integrals in Eqs. (56) and (57). In Appendix Q , these two integrals are evaluated in saddle-point approximation in the large- $N$ limit. Again, $f_{1}(t) f_{2}(t)$ is of order $1 / N$ for $t<t_{c}$ and suppressed exponentially for $t>t_{c}$, respectively, where now

$$
t_{c}=\frac{1}{\Sigma^{2}} \frac{1}{N} \sum_{n=1}^{N} \frac{1}{y_{n}^{2}} .
$$

Note that $t_{c} \geq 1$ by $(\sqrt[66]{ })$ and that $t_{c}$ is infinite if some of the $y_{n}$ are zero. Again, only the interval $1 \leq t \leq t_{c}$ contributes to leading order in $1 / N$, and we obtain from Eqs. (B.8) and (B.15) using Eqs. (C.8) and (C.9)

$$
f_{1}(t) f_{2}(t)=\frac{\pi}{N} J_{0}\left(\Sigma^{2} u_{p} \bar{x}\right) J_{0}\left(\Sigma^{2} u_{q} \bar{x}\right) \frac{\bar{x}^{2}}{\mathcal{L}_{2}^{\prime \prime}(\bar{x})}
$$

to leading order in $1 / N$ in this region of $t$, where $\mathcal{L}_{2}^{\prime \prime}(\bar{x})$ is given by Eq. (C.6) and $\bar{x}$ is implicitly given by Eq. (70) below. To proceed, we substitute $z=\Sigma^{2} \bar{x}$ and obtain

$$
d z=\Sigma^{2} \frac{d \bar{x}}{d t} d t
$$


To compute $d \bar{x} / d t$, we use the saddle-point equation (C.2) in the form

$$
\Sigma^{2} t=\frac{1}{N} \sum_{n=1}^{N} \frac{1}{y_{n}^{2}+\bar{x}^{2}}
$$

and differentiate with respect to $t$ to obtain

$$
\Sigma^{2}=-2 \bar{x} \frac{1}{N} \sum_{n=1}^{N} \frac{1}{\left(y_{n}^{2}+\bar{x}^{2}\right)^{2}} \frac{d \bar{x}}{d t}=-\frac{1}{2 \bar{x}} \mathcal{L}_{2}^{\prime \prime}(\bar{x}) \frac{d \bar{x}}{d t},
$$

where we have used Eq. (C.6) in the last equality. Simple algebra yields

$$
\frac{\bar{x}^{2}}{\mathcal{L}_{2}^{\prime \prime}(\bar{x})} d t=-\frac{1}{2 \Sigma^{6}} z d z
$$

so that Eq. (55) in connection with Eq. (68) reduces to Eq. (62) with $\Xi=$ $\Sigma^{2} \bar{x}(t=1)$, where $\bar{x}(t=1)$ is the solution of $(70)$ for $t=1$. We have thus reduced the general case to the special case considered in the previous subsection. The only complication in the general case is that one has to solve the higher-order polynomial equation $(70)$ for $\bar{x}(t=1)$. This is no problem numerically since we have shown in Appendix 9 that there is only one real and positive solution which has to be determined.

\section{Discussion}

We have considered a random matrix model appropriate for the Dirac operator of QCD at finite temperature and derived the spectral density, the universal spectral correlations on the scale of the mean level spacing, and the microscopic limits of the spectral density and spectral correlation functions. The calculation was done for an arbitrary real diagonal matrix added to the random matrix of the Dirac operator. The fact that the functional form of the microscopic correlations remains unchanged even with an arbitrary deterministic matrix added adds further evidence to the conjecture that these correlations (and in particular the microscopic spectral density) are universal.

It is a well known feature of standard random matrix theory that many of the conceptual and formal aspects are common to all three ensembles. In particular, the calculation of fluctuations shows these common features since the scale is always set by the mean level spacing. Therefore, we conjecture that the universal scaling behavior which we derived for all correlations in the chGUE carries over to the chGOE and the chGSE. More precisely, we expect that, after proper rescaling with the chiral condensate, the microscopic spectral correlations are given by the known zero-temperature results for the chGOE and the chGSE, respectively [5, 6]. 
There are two important directions in which one would like to generalize the results of this paper. First, one would like to extend our results to a nonzero number of flavors and non-zero topological charge. This presents certain technical challenges to the graded eigenvalue method, but we are convinced that an application of the Itzykson-Zuber integral for rectangular supermatrices [32 will solve this problem. The result, however, can be conjectured now: After proper rescaling with the chiral condensate, we will obtain the same dependence on $N_{f}$ and $\nu$ as in the zero-temperature result computed in Ref. 沟. Second, one would like to consider complex diagonal matrices instead of just real ones in order to model the effect of the chemical potential on the spectrum of the Dirac operator. For the latter case, it is important to go to $N_{f} \neq 0$, i.e., to the unquenched case, as was argued by Stephanov [17]. At present, there is quite some effort in this direction [39].

Note: After completion of the present work, we learned that a similar calculation was performed simultaneously and independently by Jackson, Şener, and Verbaarschot.

\section{Acknowledgments}

We would like to thank A. Müller-Groeling, A. Schäfer, J.J.M. Verbaarschot, and H.A. Weidenmüller for useful discussions.

\section{A Uniform convergence}

The integral over $\psi$ in Eq. (25) does not converge uniformly since the matrix $\sigma$ is arbitrary complex rather than hermitian so that its eigenvalues are complex rather than real. Note that it is sufficient to consider only the commuting variables of $\sigma$ since the anticommuting variables cannot cause convergence problems. Hence, the $\sigma$ - and the $\psi$-integration in (25) cannot simply be interchanged.

The obvious way to proceed is to deform the integration contour of the $\sigma$ variables so that uniform convergence is obtained. This was done in Ref. [19] where the one-point function was computed by an explicit re-parameterization of the $\sigma$-variables. This entails replacing compact integration variables by non-compact ones. The generalization of this approach to higher correlation functions is not trivial. One would have to pseudo-diagonalize $\sigma$ using noncompact groups and compute the resulting integrals of the Itzykson-Zuber type. 
However, there is an easier way to proceed. In the calculation of the one-point function, one observes that a naive interchange of the $\sigma$ - and $\psi$-integrations, while upsetting the real part of the result, leaves the imaginary part of the result intact [19]. The imaginary part of the result is all that is needed to construct the one-point function. Hence, as far as the one-point function is concerned, we can interchange the $\sigma$ - and $\psi$-integrations and still get the correct result. While there is no proof at present that this remarkable feature persists for higher correlation functions, we conjecture that it does. This conjecture is very strongly supported by the fact that we obtain the correct zero-temperature result for the higher correlation functions by simply interchanging the order of the integrations as we have done in the text. Since the convergence problem is completely unrelated to the introduction of the matrix $Y$ at finite temperature, it is hard to see how the presence of $Y$ could change anything in this respect.

Of course, this problem deserves further attention. Work in this direction is in progress, but there is no doubt that the results presented in this paper will be unaffected by a clarification of this issue.

\section{B Saddle-point approximation for the special case}

We wish to compute the two integrals in Eqs. (58) and (59) in the large- $N$ limit by saddle-point approximation. An interesting phenomenon occurs since the integrals depend on an external parameter $t$. We shall see that the integrals can be of different order in $1 / N$ depending on the value of $t$.

Let us start with the integral in Eq. (59) since it is conceptually simpler. Combining terms which grow exponentially with $N$, we obtain

$$
\begin{aligned}
f_{2}(t) & =\int_{0}^{\infty} d s_{2} s_{2} J_{0}\left(\Sigma^{2} u_{q} s_{2}\right) \exp \left(-N \mathcal{L}_{2}\left(s_{2}\right)\right) \\
\mathcal{L}_{2}\left(s_{2}\right) & =\Sigma^{2} t s_{2}^{2}-\log \left(y^{2}+s_{2}^{2}\right) .
\end{aligned}
$$

To minimize $\mathcal{L}_{2}\left(s_{2}\right)$, we differentiate

$$
\frac{\partial \mathcal{L}_{2}}{\partial s_{2}}=2 \Sigma^{2} t s_{2}-\frac{2 s_{2}}{y^{2}+s_{2}^{2}} \stackrel{!}{=} 0
$$

There are three possible solutions for $s_{2}, \bar{s}_{2}=0$ and $\bar{s}_{2}= \pm \bar{x}$ with

$$
\bar{x}=\frac{1}{\Sigma} \sqrt{\frac{1}{t}-\frac{1}{t_{c}}},
$$


where $t_{c}=1 /(\Sigma y)^{2}$. Expanding about the saddle points yields

$$
\mathcal{L}_{2}\left(\bar{s}_{2}+\delta s_{2}\right)=\mathcal{L}_{2}\left(\bar{s}_{2}\right)+\frac{1}{2} \mathcal{L}_{2}^{\prime \prime}\left(\bar{s}_{2}\right)\left(\delta s_{2}\right)^{2}
$$

with

$$
\begin{array}{ll}
\mathcal{L}_{2}(0)=-\log \left(y^{2}\right) & \mathcal{L}_{2}( \pm \bar{x})=1-t / t_{c}+\log \left(\Sigma^{2} t\right) \\
\mathcal{L}_{2}^{\prime \prime}(0)=2 \Sigma^{2}\left(t-t_{c}\right) & \mathcal{L}_{2}^{\prime \prime}( \pm \bar{x})=4 \Sigma^{2} t\left(1-t / t_{c}\right) .
\end{array}
$$

The sign of $\mathcal{L}_{2}^{\prime \prime}$ determines the location of the minimum of $\mathcal{L}_{2}$. For $t>t_{c}$, the minimum is at 0 . For $t<t_{c}$, the minimum is at $\bar{x}$, and $\bar{x}$ is real. The solution $-\bar{x}$ lies outside the interval of integration and can be discarded. We now have to integrate over the fluctuations. For $\bar{s}_{2}=\bar{x}$, we obtain to leading order in $1 / N$

$$
\begin{aligned}
f_{2}(t) & =\bar{x} J_{0}\left(\Sigma^{2} u_{q} \bar{x}\right) e^{-N \mathcal{L}_{2}(\bar{x})} \int_{-\infty}^{\infty} d\left(\delta s_{2}\right) e^{-\frac{N}{2} \mathcal{L}_{2}^{\prime \prime}(\bar{x})\left(\delta s_{2}\right)^{2}} \\
& =\sqrt{\frac{2 \pi}{N}} e^{-N \mathcal{L}_{2}(\bar{x})} J_{0}\left(\Sigma^{2} u_{q} \bar{x}\right) \frac{\bar{x}}{\sqrt{\mathcal{L}_{2}^{\prime \prime}(\bar{x})}} \quad \text { for } t<t_{c}
\end{aligned}
$$

For $\bar{s}_{2}=0$, we obtain to leading order in $1 / N$

$$
\begin{aligned}
f_{2}(t) & =e^{-N \mathcal{L}_{2}(0)} \int_{0}^{\infty} d\left(\delta s_{2}\right) \delta s_{2} e^{-\frac{N}{2} \mathcal{L}_{2}^{\prime \prime}(0)\left(\delta s_{2}\right)^{2}} \\
& =\frac{1}{N} \frac{e^{-N \mathcal{L}_{2}(0)}}{\mathcal{L}_{2}^{\prime \prime}(0)} \quad \text { for } \quad t>t_{c} .
\end{aligned}
$$

Now consider the integral in Eq. (58). We again combine terms which grow exponentially with $N$ to obtain

$$
\begin{aligned}
f_{1}(t) & =\operatorname{Im} \int_{0}^{\infty} d s_{1} s_{1} I_{0}\left(\Sigma^{2} u_{p} s_{1}\right) \exp \left(-N \mathcal{L}_{1}\left(s_{1}\right)\right) \\
\mathcal{L}_{1}\left(s_{1}\right) & =\Sigma^{2} t s_{1}^{2}+\log \left(y^{2}-s_{1}^{2}\right),
\end{aligned}
$$

where we have omitted the imaginary increment of $s_{1}$ in $\mathcal{L}_{1}$. Differentiation yields

$$
\frac{\partial \mathcal{L}_{1}}{\partial s_{1}}=2 \Sigma^{2} t s_{1}-\frac{2 s_{1}}{y^{2}-s_{1}^{2}} \stackrel{!}{=} 0
$$


This equation is identical to Eq. (B.3) if $s_{1}$ is replaced by $i s_{2}$. Hence, possible solutions are $\bar{s}_{1}=0$ and $\bar{s}_{1}= \pm i \bar{x}$. Expansion about the saddle points yields

$$
\begin{array}{ll}
\mathcal{L}_{1}(0)=\log \left(y^{2}\right) & \mathcal{L}_{1}( \pm i \bar{x})=t / t_{c}-1-\log \left(\Sigma^{2} t\right) \\
\mathcal{L}_{1}^{\prime \prime}(0)=2 \Sigma^{2}\left(t-t_{c}\right) & \mathcal{L}_{1}^{\prime \prime}( \pm i \bar{x})=4 \Sigma^{2} t\left(1-t / t_{c}\right) .
\end{array}
$$

Note that $\mathcal{L}_{1}\left(i \bar{s}_{2}\right)=-\mathcal{L}_{2}\left(\bar{s}_{2}\right)$. Although the saddle-point equations (B.3) and (B.12) look remarkably similar, the structure of the saddle points is now quite different. In the following, we show that the saddle point at $i \bar{x}(-i \bar{x})$ makes the dominant contribution for $t<t_{c}\left(t>t_{c}\right)$.

Consider first the case $t<t_{c}$ where $\bar{x}>0$. The possible saddle points $\pm i \bar{x}$ are purely imaginary. Hence, we have to deform the contour of integration into the complex plane to reach these saddle points. Due to the positive sign of the imaginary increment of $s_{1}$, only the point $+i \bar{x}$ can be reached. Since $\mathcal{L}_{1}^{\prime \prime}(i \bar{x})$ is positive for $t<t_{c}$, we have to integrate over quadratic fluctuations parallel to the real axis. Since the original range of integration was 0 to $\infty$ and since the saddle point lies on the imaginary axis, the integration over the fluctuations is only from 0 to $\infty$. We obtain for the contribution from the saddle point $i \bar{x}$

$$
\begin{aligned}
f_{1}(t) & =\operatorname{Im} i \bar{x} I_{0}\left(\Sigma^{2} u_{p} i \bar{x}\right) e^{-N \mathcal{L}_{1}(i \bar{x})} \int_{0}^{\infty} d\left(\delta s_{1}\right) e^{-\frac{N}{2} \mathcal{L}_{1}^{\prime \prime}(i \bar{x})\left(\delta s_{1}\right)^{2}} \\
& =\sqrt{\frac{\pi}{2 N}} e^{-N \mathcal{L}_{1}(i \bar{x})} J_{0}\left(\Sigma^{2} u_{p} \bar{x}\right) \frac{\bar{x}}{\sqrt{\mathcal{L}_{1}^{\prime \prime}(i \bar{x})}} \quad \text { for } t<t_{c} .
\end{aligned}
$$

The saddle point $\bar{s}_{1}=0$ does not contribute at all since the imaginary part of the product of the value of the integrand at the saddle point and the integral over the fluctuations is zero in this case.

Now consider the case $t>t_{c}$ where $\bar{x}$ is imaginary. The relevant saddle point is now $\bar{s}_{1}=-i \bar{x}$ which is real and positive. The point $i \bar{x}$ is real and negative and, hence, outside the interval of integration. Since $\mathcal{L}_{1}^{\prime \prime}(-i \bar{x})$ is negative for $t>t_{c}$, we now have to integrate over quadratic fluctuations parallel to the imaginary axis from 0 to $\infty$. We obtain

$$
\begin{aligned}
f_{1}(t) & =\operatorname{Im}(-i \bar{x}) I_{0}\left(-\Sigma^{2} u_{p} i \bar{x}\right) e^{-N \mathcal{L}_{1}(-i \bar{x})} \int_{0}^{\infty} d\left(i \delta s_{1}\right) e^{-\frac{N}{2} \mathcal{L}_{1}^{\prime \prime}(-i \bar{x})\left(i \delta s_{1}\right)^{2}} \\
& =\sqrt{\frac{\pi}{2 N}} e^{-N \mathcal{L}_{1}(-i \bar{x})} J_{0}\left(\Sigma^{2} u_{p} \bar{x}\right) \frac{-i \bar{x}}{\sqrt{-\mathcal{L}_{1}^{\prime \prime}(-i \bar{x})}} \quad \text { for } \quad t>t_{c}
\end{aligned}
$$

which is identical in structure with Eq. (B.15). Again, the saddle point $\bar{s}_{1}=0$ does not contribute for the same reason as in the case $t<t_{c}$. 
Combining Eqs. (B.8) and (B.15) we obtain Eq. (61) which is valid for $t<t_{c}$. Hence, $f_{1}(t) f_{2}(t) \sim 1 / N$ in this region of $t$. For $t>t_{c}$, Eqs. (B.9) and (B.16) combine to yield

$$
f_{1}(t) f_{2}(t)=\frac{\sqrt{\pi}}{\sqrt{32} N^{3 / 2} \Sigma^{4} t t_{c}} I_{0}\left(\Sigma u_{p} \sqrt{1 / t_{c}-1 / t}\right) \frac{e^{-N\left(\frac{t}{t_{c}}-1-\log \frac{t}{t_{c}}\right)}}{t / t_{c}-1}
$$

showing that $f_{1}(t) f_{2}(t)$ is suppressed exponentially in this case.

We would like to add that we have checked Eqs. (B.15) and (B.16) as well as the corresponding results of Appendix $\mathrm{C}$ by an independent finite- $N$ calculation and taking the limit $N \rightarrow \infty$.

\section{Saddle-point approximation for the general case}

We now compute the more general integrals in Eqs. (56) and (57) in the large- $N$ limit by saddle-point approximation. We shall encounter the same phenomena as in Appendix B. For simplicity, we restrict ourselves to the case where all $y_{n}$ are different and non-zero as in Sec.4.2. It is straightforward to modify our arguments to work for special cases (some of the $y_{n}$ are equal or zero) as well.

Again, we write Eq. (57) in the form (B.2), where now

$$
\mathcal{L}_{2}\left(s_{2}\right)=\Sigma^{2} t s_{2}^{2}-\frac{1}{N} \sum_{n=1}^{N} \log \left(y_{n}^{2}+s_{2}^{2}\right) .
$$

To minimize $\mathcal{L}_{2}\left(s_{2}\right)$, we differentiate

$$
\frac{\partial \mathcal{L}_{2}}{\partial s_{2}}=2 \Sigma^{2} t s_{2}-2 s_{2} \frac{1}{N} \sum_{n=1}^{N} \frac{1}{y_{n}^{2}+s_{2}^{2}} \stackrel{!}{=} 0 .
$$

The obvious solution $\bar{s}_{2}=0$ persists. There are now many more solutions for $s_{2}^{2}$ ( $N$ of them, to be precise), but we show in the following that there is only one real and positive solution for $s_{2}^{2}$ if $t<t_{c}$ and no real and positive solution for $s_{2}^{2}$ if $t>t_{c}$, where $t_{c}$ is given by Eq. (67). Define

$$
g(x)=t-\frac{1}{\Sigma^{2} N} \sum_{n=1}^{N} \frac{1}{y_{n}^{2}+x} .
$$

We seek real and positive solutions of $g(x)=0$. We have

$$
g^{\prime}(x)=\frac{1}{\Sigma^{2} N} \sum_{n=1}^{N} \frac{1}{\left(y_{n}^{2}+x\right)^{2}}>0
$$




$$
\begin{aligned}
\lim _{x \rightarrow \infty} g(x) & =t>0 \\
g(0) & =t-t_{c}\left\{\begin{array}{lll}
<0 & \text { for } t<t_{c} \\
>0 & \text { for } & t>t_{c}
\end{array}\right.
\end{aligned}
$$

The function $g(x)$ increases monotonically for $x>0$ and is continuous in this region. It reaches a positive value as $x \rightarrow \infty$. Thus, $g(x)$ intersects the $x$-axis once and only once if $g(0)<0$ and not at all if $g(0)>0$. This proves our claim.

Let $\bar{x}$ be the only real and positive solution of $g\left(x^{2}\right)=0$ for $t<t_{c}$. Thus, $\bar{s}_{2}=\bar{x}$ is the only other candidate for a minimum of $\mathcal{L}_{2}$. Expansion about the saddle points as in Eq. (B.5) yields

$$
\begin{array}{ll}
\mathcal{L}_{2}(0)=-\frac{1}{N} \sum_{n=1}^{N} \log \left(y_{n}^{2}\right) & \mathcal{L}_{2}(\bar{x})=\Sigma^{2} t \bar{x}^{2}-\frac{1}{N} \sum_{n=1}^{N} \log \left(y_{n}^{2}+\bar{x}^{2}\right) \\
\mathcal{L}_{2}^{\prime \prime}(0)=2 \Sigma^{2}\left(t-t_{c}\right) & \mathcal{L}_{2}^{\prime \prime}(\bar{x})=4 \bar{x}^{2} \frac{1}{N} \sum_{n=1}^{N} \frac{1}{\left(y_{n}^{2}+\bar{x}^{2}\right)^{2}}
\end{array}
$$

The explicit form of $\mathcal{L}_{2}(0)$ and $\mathcal{L}_{2}(\bar{x})$ will not be needed in the following. We again obtain that the minimum of $\mathcal{L}_{2}$ is at $\bar{x}$ for $t<t_{c}$ and at 0 for $t>t_{c}$. Integration over the fluctuations yields Eqs. $(\overline{B .8})$ and $(\overline{B .9})$ as before.

Writing Eq. (56) in the form (B.11) yields

$$
\mathcal{L}_{1}\left(s_{1}\right)=\Sigma^{2} t s_{1}^{2}+\frac{1}{N} \sum_{n=1}^{N} \log \left(y_{n}^{2}-s_{1}^{2}\right) .
$$

Note that $\mathcal{L}_{1}\left(i s_{2}\right)=-\mathcal{L}_{2}\left(s_{2}\right)$. For the $s_{1}$-integration, there are now many more candidates for saddle points. Apart from the trivial solution $\bar{s}_{1}=0$ (which does not contribute for the reason discussed in Appendix B), there are $2 N$ other solutions for $\bar{s}_{1}$. Defining a function similar to $g(x)$ and investigating its pole structure, we find that for $t<t_{c}$ there are $N-1$ real and positive solutions for $\bar{s}_{1}^{2}$. None of these corresponds to a minimum of $\mathcal{L}_{1}$ on the real axis since $\mathcal{L}_{1}^{\prime \prime}<0$ at these points. The remaining two solutions are $\bar{s}_{1}= \pm i \bar{x}$, and expansion about the saddle points yields

$$
\begin{aligned}
& \mathcal{L}_{1}( \pm i \bar{x})=-\mathcal{L}_{2}(\bar{x}) \\
& \mathcal{L}_{1}^{\prime \prime}( \pm i \bar{x})=\mathcal{L}_{2}^{\prime \prime}(\bar{x}) .
\end{aligned}
$$

As in Appendix $\mathrm{B}$, the relevant saddle point is $\bar{s}_{1}=i \bar{x}$, and integration over the fluctuations yields Eqs. (B.15) as before. Eqs. (B.8) and (B.15) combine to yield Eq. (68). 
For $t>t_{c}$, there are $N$ real solutions for $\bar{s}_{1}^{2}$ (apart from $\bar{s}_{1}=0$ ). The negative solution for $\bar{s}_{1}^{2}$ for $t<t_{c}$ has now becomes positive, and it is straightforward to see from the structure of the function $g(x)$ that it is now the smallest of all possible solutions for $\bar{s}_{1}^{2}$. Note that we still have $\mathcal{L}_{1}^{\prime \prime}<0$ at all possible saddle points. We now deform the integration contour as in Appendix B: We move on the real axis from 0 to the smallest positive saddle point and then up parallel to the imaginary axis. Only the smallest positive saddle point contributes to $f_{1}(t)$, and we obtain a result similar to Eq. (B.16), with $-i \bar{x}$ replaced by the smallest positive saddle point. The product $f_{1}(t) f_{2}(t)$ is again suppressed exponentially for $t>t_{c}$ so that only the interval $1 \leq t \leq t_{c}$ contributes to the integration over $t$ in Eq. (55).

\section{References}

[1] M.A. Nowak, J.J.M. Verbaarschot, and I. Zahed, Phys. Lett. B 217 (1989) 157.

[2] Yu.A. Simonov, Phys. Rev. D 43 (1991) 3534.

[3] E.V. Shuryak and J.J.M. Verbaarschot, Nucl. Phys. A 560 (1993) 306.

[4] J.J.M. Verbaarschot and I. Zahed, Phys. Rev. Lett. 70 (1993) 3852.

[5] J.J.M. Verbaarschot, Nucl. Phys. B 426 [FS] (1994) 559.

[6] T. Nagao and K. Slevin, J. Math. Phys. 34 (1993) 2075; T. Nagao and P.J. Forrester, Nucl. Phys. B 435 (1995) 401;

[7] J.J.M. Verbaarschot, Phys. Rev. Lett. 72 (1994) 2531.

[8] J.J.M. Verbaarschot, Acta Phys. Pol. B 25 (1994) 133; Nucl. Phys. B 427 (1994) 534.

[9] J.J.M. Verbaarschot and I. Zahed, Phys. Rev. Lett. 73 (1994) 2288.

[10] M.A. Halasz and J.J.M. Verbaarschot, Phys. Rev. Lett. 74 (1995) 3920; M.A. Halasz, T. Kalkreuter, and J.J.M. Verbaarschot, Nucl. Phys. B (Proc. Suppl.) 53 (1997) 266.

[11] A. Smilga and J.J.M. Verbaarschot, Phys. Rev. D 51 (1995) 829.

[12] M.A. Halasz and J.J.M. Verbaarschot, Phys. Rev. D 52 (1995) 2563.

[13] J.J.M. Verbaarschot, Phys. Lett. B 368 (1996) 137.

[14] A.D. Jackson and J.J.M. Verbaarschot, Phys. Rev. D 53 (1996) 7223.

[15] T. Wettig, A. Schäfer, and H.A. Weidenmüller, Phys. Lett. B 367 (1996) 28.

[16] M.A. Stephanov, Phys. Lett. B 375 (1996) 249.

[17] M.A. Stephanov, Phys. Rev. Lett. 76 (1996) 4472. 
[18] J. Jurkiewicz, M.A. Nowak, and I. Zahed, Nucl. Phys. B 478 (1996) 605; M.A. Nowak, G. Papp, and I. Zahed, Phys. Lett. B 389 (1996) 137, 341; R.A. Janik, M.A. Nowak, and I. Zahed, Phys. Lett. B 392 (1997) 155.

[19] A.D. Jackson, M.K. Şener, and J.J.M. Verbaarschot, Nucl. Phys. B 479 (1996) 707.

[20] A.D. Jackson, M.K. Şener, and J.J.M. Verbaarschot, Phys. Lett. B 387 (1996) 355.

[21] J.J.M. Verbaarschot, hep-lat/9606009; Nucl. Phys. (Proc. Suppl.) 53 (1997) 88.

[22] O. Bohigas and M.J. Giannoni, Lec. Not. Phys. 209 (Springer, Heidelberg, 1984).

[23] M.L. Mehta, Random Matrices, 2nd ed. (Academic Press, New York, 1991).

[24] F. Haake, Quantum Signatures of Chaos (Springer, Berlin, 1991).

[25] T. Banks and A. Casher, Nucl. Phys. B 169 (1980) 103.

[26] H. Leutwyler and A. Smilga, Phys. Rev. D 46 (1992) 5607.

[27] K. Slevin and T. Nagao, Phys. Rev. Lett. 70 (1993) 635.

[28] E. Brézin, S. Hikami, and A. Zee, Nucl. Phys. B 464 (1996) 411; S. Nishigaki, Phys. Lett. B 387 (1996) 139.

[29] G. Akemann, P.H. Damgaard, U. Magnea, and S. Nishigaki, Nucl. Phys. B 487 (1997) 721.

[30] T. Wettig, T. Guhr, A. Schäfer, and H.A. Weidenmüller, hep-ph/9701387.

[31] F.J. Dyson, J. Math. Phys. 3 (1962) 140, 157, 166, and 1199.

[32] T. Guhr and T. Wettig, J. Math. Phys. 37 (1996) 6395.

[33] E.P. Wigner, Ann. Math. 53 (1951) 36.

[34] K.B. Efetov, Adv. Phys. 32 (1983) 53.

[35] J.J.M. Verbaarschot, H.A. Weidenmüller, and M. Zirnbauer, Phys. Rep. 129 (1985) 367.

[36] T. Guhr, Phys. Rev. Lett. 76 (1996) 2258; Ann. Phys. (NY) 250 (1996) 145.

[37] T. Guhr, J. Math. Phys. 32 (1991) 336.

[38] M. Abramowitz and I.A. Stegun, Handbook of Mathematical Functions (Dover, New York, 1972).

[39] see, e.g., R.A. Janik, M.A. Nowak, G. Papp, and I. Zahed, Phys. Rev. Lett. 77 (1996) 4876; R.A. Janik, M.A. Nowak, G. Papp, J. Wambach, and I. Zahed, hep-ph/9609491; J. Feinberg and A. Zee, cond-mat/9703087; M.A. Halasz, A.D. Jackson, and J.J.M. Verbaarschot, hep-lat/9703006. 